\title{
Intervenções do professor e a aprendizagem de matemática na Cultura Digital: a proposta pedagógica - uma experiência no $9^{\circ}$ ano
}

\author{
Eduardo Britto Velho de Mattos - CAp/UFRGS - eduardo.britto@ufrgs.br \\ Marcus Vinicius de Azevedo Basso - IME-PGEMat-PGIE/UFRGS - mbasso@ufrgs.br \\ Léa da Cruz Fagundes - LEC-PGIE/UFRGS - leafagun@ufrgs.com.br
}

Resumo: O presente estudo é fruto da investigação sobre intervenções do professor que promovam aprendizagens de matemática na Cultura Digital a partir de Projetos de Aprendizagem desenvolvidos por estudantes da Educação Básica. Discute-se uma importante perspectiva de intervenção, que é a proposta pedagógica desenvolvida com os estudantes, a qual denominamos intervenção do tipo 1. Busca-se subsídio na Epistemologia Genética piagetiana para discutir os processos de aprendizagem e em conceitos da Cultura Digital para analisar as mudanças promovidas pelas tecnologias digitais e projetar a estruturação de uma escola da Cultura Digital. Com base na experiência desenvolvida com estudantes da escola pública, analisa-se a viabilidade da proposta e as construções conceituais de matemática possibilitadas.

Palavras-Chaves: Intervenção do Professor; Projetos de Aprendizagem; Aprendizagem de Matemática; Cultura Digital

\section{Teacher interventions and mathematics learning in Digital Culture: the pedagogical proposal - an experience in 9th grade}

\begin{abstract}
The present study is the result of research on teacher interventions that promote learning of mathematics in Digital Culture from Learning Projects developed by students of Basic Education. An important intervention perspective is discussed, which is the pedagogical proposal developed with the students, which we call type 1 intervention. We seek a subsidy in Piagetian Genetic Epistemology to discuss learning processes and concepts of Digital Culture, to analyze the changes promoted by digital technologies and to design the structuring of a Digital Culture school. Based on the experience realized with public school students, the viability of the proposal and the conceptual constructions of mathematics are analyzed.
\end{abstract}

Keywords: Teacher Intervention; Learning Projects; Mathematics Learning; Digital Culture.

\section{INTRODUÇÃO}

Conversar sobre educação em um ambiente social ou mesmo acadêmico, iniciar um debate a respeito de mudanças necessárias à escola, propor comparações entre o estudante de hoje e o de outras gerações ou simplesmente iniciar uma discussão sobre aprender matemática na escola... Inevitavelmente esses são assuntos intensos e que não se esgotam com soluções prontas e incontestáveis. Há uma infinidade de opiniões, desde as centradas em experiências pessoais até as baseadas em estudos específicos e 
investigações em educação. Observa-se que a grande maioria das pessoas têm fortes conclusões em relação aos caminhos que a escola deve seguir.

Tratando-se da aprendizagem de matemática, a contraposição de ideias costuma ser enorme. Variadas são as práticas e muitos os debates, há propostas que defendem o predomínio do conteúdo sobre o fazer pedagógico, desconsiderando contextos sociais ou culturais. Outras, porém, parecem focar apenas em novas técnicas e procedimentos, sem evidenciar preocupação em promover aprendizagens conceituais de matemática.

Levando em consideração esse contexto de mudanças e constantes questionamentos e debates sobre a Escola, pretendemos desenvolver uma análise sobre o estudante e a construção de conceitos de matemática, dentro de uma perspectiva de educação que priorize a aprendizagem do estudante como função central da escola. Nessa perspectiva, é imperioso iniciar qualquer análise ou prospecção pelo jovem aprendiz, suas necessidades, possibilidades e desejos.

Conforme indicações de Veen e Vrakking (2009), os jovens da geração atual (denominados Homo zappiens, pelos autores), cresceram com o mouse e o controle remoto nas mãos, descobriram o mundo por meio de uma grande variedade de canais de televisão, jogos de computador, sites, redes sociais e telefones celulares. As implicações disso no modo pelo qual a sociedade se constitui e se organiza são enormes. Observa-se que os jovens de hoje, sujeitos da Cultura Digital, não veem mais sentido no modo como as escolas atuam em suas vidas. As sequências curriculares, as tarefas, o uso da tecnologia e as possibilidades de interação e comunicação, assim como as fontes de informação acessadas, são muito pobres e artificiais para eles.

A partir desse contexto brevemente exposto, propomos uma investigação sobre intervenções do professor que sejam promotoras de aprendizagens de matemática na Cultura Digital, tendo como perspectiva principal tratar da seguinte questão: Quais categorias de intervenção do professor devem subsidiar a ação pedagógica com estudantes dos anos finais do ensino fundamental para a promoção da aprendizagem de matemática na Cultura Digital a partir de Projetos de Aprendizagem?

Inspiradas nessa questão, e como forma de traçar a trajetória de pesquisa que será seguida, levantamos as seguintes questões complementares:

i) Como o jovem da Cultura Digital aprende?

ii) $\mathrm{O}$ desenvolvimento de Projetos de Aprendizagem é uma proposta promotora de aprendizagens e adequada à concepção de uma escola da Cultura Digital?

iii) É possível estruturar e desenvolver uma organização curricular de matemática em sistemas conceituais para os anos finais do ensino fundamental a partir de Projetos de Aprendizagem?

iv) Quais princípios devem ser observados pelo professor como condição necessária para que a sua intervenção promova construções de conceitos de matemática a partir do desenvolvimento de Projetos de Aprendizagem por estudantes dos anos finais do ensino fundamental?

No presente artigo, pretendemos iniciar a análise relativa às questões (i) e (ii), a partir de um estudo teórico sobre a aprendizagem dos jovens estudantes, sujeitos da Cultura Digital, e de uma experiência realizada com estudantes do $9^{\circ}$ ano do ensino fundamental de uma escola pública de Porto Alegre. 


\section{A APRENDIZAGEM NA CULTURA DIGITAL}

Inicialmente é importante observar que, de acordo com Rosa (2013, p.43), a Cultura Digital é "marcada pelo uso de tecnologias digitais e possui como diferencial o estabelecimento de uma nova relação com o real, que se estratifica através de novas possibilidades de comunicação e representação". A autora, complementando-se com Basso (2003), indica que as tecnologias digitais favorecem surpreendentes representações do espaço, tais como a holografia, a realidade virtual, estereogramas e fractais, possibilitando a combinação de diversas dimensões: luz, som, movimento, tempo, espaço. Assim, as tecnologias digitais permitem ao ser humano expandir sua capacidade de abstração. Especificamente, demonstram que a Cultura Digital corresponde a uma expansão dos poderes cognitivos da mente.

A apropriação das tecnologias digitais e o uso da internet se tornam coletivos, de modo que para as novas gerações é natural tê-los a disposição e usá-los livremente. Produzir informação coletivamente, por meio da colaboração e da cooperação, e tornála disponível é uma marca que caracteriza fortemente a Cultura Digital (LEMOS, 2002).

Há, então, importantes transformações na sociedade: cognitivamente o ser humano tem as suas capacidades ampliadas; em termos da produção de informação e conhecimento, há uma mudança no modo como se constrói, distribui e acessa, sendo priorizado o coletivo, a colaboração e a cooperação; e em relação à comunicação, ela é potencializada com o advento da internet.

Considerando apenas esses três aspectos, é imediato inferir que o jovem da Cultura Digital é diferente. Organicamente, trata-se da mesma espécie, no entanto esse jovem é um novo sujeito, imerso em uma nova cultura, que possibilita diferentes interações e experiências. Biológica e psicologicamente os mecanismos e processos de aprendizagem são preservados, mas cabe questionar quais mudanças a Cultura Digital promove no modo pelo qual se aprende e, consequentemente, no modo pelo qual uma escola da Cultura Digital deve se estruturar. Antes de discutir as possíveis mudanças advindas da Cultura Digital, precisamos buscar subsídios que exponham e expliquem os processos de aprendizagem. A nossa opção teórica encontra na Epistemologia Genética, desenvolvida por Jean Piaget, esses fundamentos, os quais serão discutidos a seguir.

\subsection{Uma perspectiva piagetiana para a aprendizagem na escola}

A Epistemologia Genética é composta por uma vasta produção de Piaget a partir do seu desejo de compreender como se dão os processos de aquisição de conhecimento, com especial interesse em estudar a passagem de um estágio de menos conhecimento para um estágio de mais conhecimento. Ainda que Piaget não tenha dedicado seus trabalhos a discussões pedagógicas ou à proposição de métodos educacionais, sua contribuição à Educação é imediata, de modo que, com base em sua obra, é possível construir um conjunto de princípios sobre os quais deve se estruturar uma proposta pedagógica construtivista.

Dentre os muitos conceitos da investigação piagetiana, neste estudo decidimos eleger a Equilibração, o Fazer e Compreender e a Tomada de Consciência como conceitos estruturantes da construção do conhecimento, pelo seguinte:

- $\mathrm{Na}$ Equilibração das Estruturas Cognitivas, Piaget demonstra a perturbação cognitiva, como um conflito ou uma contradição que se apresenta como o 
obstáculo que desencadeia o processo construtivo do conhecimento, o qual é resultado das interações sujeito/objeto e do processo de equilibração majorante;

- As relações entre o Fazer e o Compreender, explicam a importância da ação e da interação do estudante na construção das suas aprendizagens, indicando o papel dos conhecimentos autônomos como ponto de partida desse processo; e

- A Tomada de Consciência é considerada como o processo de conceituação que permite ao sujeito a transição do fazer ao compreender, portanto definitivo para a aprendizagem e para o desenvolvimento cognitivo do sujeito.

Com base nessas proposições e demonstrações de Piaget, estudando seus fundamentos e propondo um diálogo com a aprendizagem escolar, elaboramos um conjunto de princípios que subsidiam a construção de propostas pedagógicas construtivistas. A seguir apresentaremos uma síntese desses princípios. Antes disso, é relevante observar, porém, que a obra de Piaget, e mesmo esse recorte que propusemos, permite outras variadas leituras e análises que podem ser agregadas às nossas e constituirão importantes implicações para o fazer pedagógico do professor.

Dessa forma, uma análise com fins pedagógicos em relação aos estudos sobre a Equilibração das Estruturas Cognitivas (PIAGET, 1976), permitem indicar sinteticamente que a ação pedagógica deve permitir ao estudante enfrentar situações que provoquem desequilíbrios cognitivos, dosados de modo sistemático, com vista a estabelecer novas relações entre os conceitos estudados. É fundamental que se considerem as construções prévias do estudante, suas estruturas cognitivas e contextos de interesse, com propostas de investigação que surjam dos seus desejos e desafios pessoais, permitindo que o estudante proponha questões para sua investigação. A intervenção do professor deve buscar, portanto, conhecer as construções cognitivas do estudante e planejar desafios que sejam subjetivamente perturbadores e levem a construção de novidades.

Ao tratar das relações entre o Fazer e o Compreender, Piaget (1978) destaca a importância da Tomada de Consciência (PIAGET, 1977) e a apresenta sob uma nova e importante perspectiva: como o caminho que leva do saber fazer à compreensão e conceituação por parte do sujeito. Em outras palavras, Piaget reapresenta a Tomada de Consciência como o processo que transforma um sucesso prático (e inconsciente) em uma aprendizagem.

Nessa perspectiva, levanta-se que uma ação pedagógica construtivista que pretenda promover a aprendizagem e o desenvolvimento cognitivo, deve possibilitar que os estudantes partam do seu real subjetivo em direção às suas necessidades de compreensão, construindo uma trajetória na qual a ação seja permeada de escolhas conscientes e pelo estabelecimento de relações entre diferentes ações, coordenando-as na busca da compreensão das razões dos sucessos e de possíveis variações. Com isso, espera-se que os estudantes superem o campo das observações de ações próprias na perspectiva das necessidades dedutivas. É fundamental que se inicie pelos conhecimentos autônomos a respeito do objeto e que as tomadas de consciência que levam a conceituação sejam fruto da problematização desses conhecimentos. A intervenção do professor deve, considerando os conhecimentos autônomos do estudante, criar situações que transformem o que era suficiente para o sujeito em determinado nível em uma contradição, um conflito ou uma lacuna, fazendo emergir novas necessidades cognitivas. 


\subsection{Uma escola da Cultura Digital}

Com o objetivo de dar continuidade ao levantamento de princípios sobre os quais acreditamos que deve ser estruturada uma proposta pedagógica construtivista e a intervenção do professor, pretende-se, nesse momento, considerar as características do jovem estudante da geração atual, sujeito da Cultura Digital, para apontar critérios que permitam a constituição uma escola da Cultura Digital.

Utilizamos a expressão escola da Cultura Digital (e não Cultura Digital na escola ou escola na Cultura Digital), com a intenção de projetar uma possível escola que se estruture com base nas modificações que as tecnologias digitais impuseram na sociedade. Diverge, então, de uma proposta educacional que se ajusta para utilizar os recursos digitais (sem negar os seus méritos), mas especialmente de uma escola que apenas integra os recursos digitais às suas práticas corriqueiras.

Arruda (2004) aponta, nesse sentido, a insuficiência da simples inserção de computadores na escola para que hajam mudanças efetivas nas estratégias e práticas pedagógicas. Indica que efetivamente são necessárias novas formas de pensar e agir diante das Tecnologias Digitais no contexto escolar.

Especificamente em relação a uma plena inclusão da escola na Cultura Digital (ou seja, a construção de uma escola da Cultura Digital), Morais (2011) e Hoffmann e Fagundes (2008), indicam fortemente a necessidade de uma verdadeira e substancial mudança de paradigma educacional.

$\mathrm{Na}$ proposição do Curso de Especialização em Educação na Cultura Digital, Ramos et al (2013, p.18-20) expressam a mesma concepção ao sugerirem a pesquisa e a investigação como princípios pedagógicos que permitem qualificar e reestruturar o processo pedagógico. Destacam, também, a importância de cada cursista ter a possibilidade de escolher os conteúdos mais adequados à sua realidade, às suas necessidades e aos seus interesses subjetivos.

Apoiando-se nessas discussões e, em especial, nas proposições de Veen e Vrakking (2009) sobre o Homo zappiens e nas construções de Mattos (2010) em relação às exigências educacionais do jovem do século XXI, indica-se que a estruturação de uma escola construtivista da Cultura Digital deve ser planejada para estudantes ativos e que podem administrar a sua aprendizagem, enfrentando situações complexas, desafiadoras e inéditas. Deve-se possibilitar um acesso criativo e inovador ao computador, à internet e aos demais recursos multimídia, priorizando a investigação do estudante e um trabalho em rede, que permitam uma visão inicial do todo, com amplas fontes de informação, e a escolha negociada dos detalhes a serem explorados pelos aprendizes. A intervenção do professor deve problematizar as descobertas e as certezas dos estudantes e fornecer subsídios para que os aprendizes avancem nas suas investigações e aprendizagens.

\subsection{Intervenções do professor - a proposta pedagógica}

Nesta investigação sobre a intervenção professor, com o objetivo da construção de um modelo de intervenção que seja promotor de aprendizagens de matemática na educação básica, diferenciamos as intervenções segundo duas perspectivas: a proposta pedagógica de modo geral (a qual denominamos intervenção do tipo 1) e a atuação docente na interação professor-aluno (intervenção do tipo 2). No presente artigo, centraremos a análise na intervenção do tipo 1 . 
Em relação ela, destaca-se a necessidade de proporcionar aos estudantes um espaço de aprender a aprender (MATTOS et al, 2016; BONA, 2012), em função da inexistência de diálogo entre a realidade do estudante e a ação em sala de aula (FREIRE, 1996; LINS, 2004); dos conteúdos serem desconexos (D’AMBROSIO, 1996); da ação dos estudantes em sala de aula ser pouco valorizada e mesmo possibilitada (BONA, 2010); da possibilidade de apropriação das tecnologias digitais em sala de aula ser muito limitada (BONA, et al, 2012), entre outros.

Como uma alternativa a esses desafios, Mattos e Basso (2011) indicam o desenvolvimento de Projetos de Aprendizagem na escola - inicialmente propostos por Fagundes, Sato e Maçada (1999) - como uma arquitetura pedagógica que atende às novas necessidades educacionais do século XXI.

A proposta de Projetos de Aprendizagem desenvolvidos por estudantes da Educação Básica, de acordo Mattos (2010, p.45), privilegia a investigação, as escolhas e a criatividade dos estudantes e, dessa forma, "revoluciona a escola, trazendo de volta a ela os alunos, seus desejos, curiosidades, espontaneidades, impulsos, criatividade". Destaca, também, que os conhecimentos disciplinares passam a ser explorados sob um novo olhar, colocando-os em relação uns com os outros e todos com a realidade, o que possibilita a sua reaproximação com o íntimo do aluno e traz novamente sentido para a existência deles (conhecimentos disciplinares).

No mesmo sentido, para que as aprendizagens de conceitos e conteúdos sejam construídas, Fagundes, Nevado e Basso (2001, p.45), apontam a necessidade de que a curiosidade do aluno, seus desejos, suas necessidades, inquietações, dúvidas e questões "decidam o assunto a ser pesquisado pois neste caso os conteúdos não serão impostos pelo professor, mas buscados pelo estudante pois a motivação para aprender é intrínseca ao indivíduo, depende de seu desejo de conhecer, de sua necessidade de saber".

O desenvolvimento de um Projeto de Aprendizagem, em resumo, pode ser caracterizado como uma investigação que tem origem nas curiosidades subjetivas do estudante e possibilita a construção interdisciplinar de conhecimentos.

Desse modo, destacamos para este estudo o desenvolvimento de Projetos de Aprendizagem como uma possibilidade de intervenção do tipo 1 compatível aos princípios levantados sobre a ação pedagógica construtivista que seja promotora de aprendizagens na Cultura Digital.

\section{UMA EXPERIÊNCIA NO $9^{\circ}$ ANO DO ENSINO FUNDAMENTAL}

Com o objetivo de analisar a proposta de desenvolvimento de Projetos de Aprendizagem por estudantes da Educação Básica enquanto promotora de aprendizagem de matemática na Cultura Digital, é importante considerar dois aspectos: a sustentação teórica e a viabilidade prática. No tópico precedente, com base nos estudos piagetianos sobre a aprendizagem, justificamos teoricamente os Projetos de Aprendizagem como uma possibilidade de intervenção do tipo 1. Neste tópico passaremos a discutir a sua viabilidade prática a partir de uma experiência realizada com estudantes do $9^{\circ}$ ano do Ensino Fundamental de uma escola pública de Porto Alegre/RS. A experiência que será relatada foi desenvolvida como estratégia pedagógica das aulas de matemática nos quatro períodos semanais destinados à disciplina, ao longo de 4 meses de atividades. Teve como público 30 jovens com idade entre 13 e 17 anos. 
Nesses períodos os estudantes formaram cinco grupos e foram desafiados a estabelecer relações e aprender matemática a partir de investigações pertinentes aos Projetos de Aprendizagem desenvolvidos por eles. Inicialmente, tendo como base os interesses e curiosidades manifestados pelos grupos, os professores da turma (o professor de matemática da escola e duas estudantes de licenciatura em matemática que colaboraram com os trabalhos ao longo de todo o ano letivo) planejaram possíveis relações com matemática e conceitos a serem construídos pelos estudantes.

Dentre as intervenções do tipo 2 desenvolvidas ao longo dos trabalhos (as quais não serão objeto de análise do presente estudo), destacamos uma que possibilitou que as aprendizagens fossem compartilhadas pela turma e acabou por se caracterizar, também, como uma intervenção do tipo 1 ao ser integrada à estratégia pedagógica desenvolvida. Em meios às investigações dos estudantes, os grupos foram desafiados a, considerando a relevância das suas descobertas e construções em matemática, planejar e desenvolver atividades que promovessem a aprendizagem dos outros grupos. Como o objetivo era de verdadeiramente promover aprendizagens, estudantes e professores combinaram que cada grupo ficaria responsável por três aulas ( 6 períodos) de atividades com a turma, as quais seriam centradas no Projeto de Aprendizagem desenvolvido pelos estudantes de cada grupo e, em especial, nas relações estabelecidas com a matemática e nos conceitos construídos.

É interessante destacar que essa intervenção permite que as aprendizagens dos estudantes construam uma rede de conceitos que estrutura um conjunto complexo de aprendizagens da turma, favorecendo o trabalho em equipe e o ato de compartilhar, características dos jovens da Cultura Digital. A perspectiva de planejar atividades que levem os colegas a aprender matemática, leva a desequilíbrios cognitivos que exigem tomadas de consciência dos processos de aprendizagem (metacognição) e favorece a busca pela compreensão dos sucessos práticos, suas possíveis variações e coordenações.

Com base no desenvolvimento das investigações e nas construções conceituais dos estudantes, organizamos a Tabela 1, de modo a facilitar a análise da viabilidade prática da proposta de Projetos de Aprendizagem como promotores de aprendizagens de matemática e, portanto, uma possibilidade de intervenção do tipo 1.

\begin{tabular}{|c|c|c|c|}
\hline Pergunta inicial & Contexto da investigação & $\begin{array}{l}\text { Relações com } \\
\text { matemática }\end{array}$ & $\begin{array}{l}\text { Conceitos de } \\
\text { matemática }\end{array}$ \\
\hline $\begin{array}{l}\text { Como as empresas } \\
\text { lucram sem que o } \\
\text { consumidor perceba? }\end{array}$ & $\begin{array}{l}\text { Os estudantes identificam } \\
\text { que através da mídia } \\
\text { capitalista e de diversos } \\
\text { outros meios, somos } \\
\text { diariamente atingidos e } \\
\text { muitas pessoas sentem a } \\
\text { obrigação de se integrarem } \\
\text { em certos padrões. }\end{array}$ & $\begin{array}{l}\text { Projeção de gastos de uma } \\
\text { família e análise dos } \\
\text { prejuízos causados por } \\
\text { possíveis endividamentos. }\end{array}$ & $\begin{array}{l}\text { Matemática financeira: } \\
\text { Juros Simples e Juros } \\
\text { Compostos; } \\
\text { Progressões Aritmética e } \\
\text { Geométrica. }\end{array}$ \\
\hline $\begin{array}{l}\text { Super-heróis: quem são? } \\
\text { Quais seus poderes? Qual } \\
\text { é o primeiro? Por que } \\
\text { usam capa? E o que tudo } \\
\text { isso tem a ver com } \\
\text { matemática? }\end{array}$ & $\begin{array}{l}\text { Os estudantes gostam muito } \\
\text { de super-heróis e no início da } \\
\text { pesquisa um novo filme do } \\
\text { Capitão América tinha } \\
\text { acabado de estrear no } \\
\text { cinema. }\end{array}$ & $\begin{array}{l}\text { Explorar alguns dos } \\
\text { superpoderes dos heróis, tais } \\
\text { como a supervelocidade e a } \\
\text { superaudição. }\end{array}$ & $\begin{array}{l}\text { Razão e Proporção (a } \\
\text { partir do cálculo de } \\
\text { velocidade média, } \\
\text { distância e tempo); } \\
\text { Função } 1^{\circ} \text { grau; } \\
\text { Noções de trigonometria } \\
\text { no triângulo retângulo e } \\
\text { teorema de Pitágoras. }\end{array}$ \\
\hline
\end{tabular}


8

\begin{tabular}{|c|c|c|c|}
\hline Pergunta inicial & Contexto da investigação & $\begin{array}{l}\text { Relações com } \\
\text { matemática }\end{array}$ & $\begin{array}{l}\text { Conceitos de } \\
\text { matemática }\end{array}$ \\
\hline $\begin{array}{l}\text { O que acontece com o } \\
\text { cadáver congelado? }\end{array}$ & $\begin{array}{l}\text { Os estudantes desejavam } \\
\text { pesquisar algo relacionado a } \\
\text { cadáveres. A partir de uma } \\
\text { piada de um dos } \\
\text { componentes do grupo sobre } \\
\text { gelo, lembraram de ouvir } \\
\text { falar sobre o congelamento } \\
\text { de corpos em busca de cura } \\
\text { de doenças com a evolução } \\
\text { da medicina e ficaram com } \\
\text { vontade de entender melhor o } \\
\text { assunto. }\end{array}$ & $\begin{array}{l}\text { Inicialmente estudar a } \\
\text { variação da temperatura de } \\
\text { um corpo - Lei de } \\
\text { resfriamento de Newton. } \\
\text { Os trabalhos do grupo } \\
\text { seguiram conforme } \\
\text { planejamento inicial, no } \\
\text { entanto, na perspectiva de } \\
\text { estudar funções exponenciais, } \\
\text { o grupo decidiu pesquisar as } \\
\text { funções mais iniciais e } \\
\text { acabou se aprofundando nas } \\
\text { funções quadráticas. Com } \\
\text { isso, também estudaram } \\
\text { equações do } 2^{\circ} \text { grau e } \\
\text { fórmula de Bhaskara. }\end{array}$ & $\begin{array}{l}\text { Construção e análise de } \\
\text { gráficos; } \\
\text { Conceito de função; } \\
\text { Noções de função } \\
\text { exponencial; } \\
\text { Função quadrática; } \\
\text { Equações do 2 } 2^{\circ} \text { grau; } \\
\text { fórmula de Bhaskara. }\end{array}$ \\
\hline $\begin{array}{l}\text { O que aconteceria se a } \\
\text { Terra atraísse outra Lua } \\
\text { para perto de si? }\end{array}$ & $\begin{array}{c}\text { Os estudantes se interessaram } \\
\text { no assunto a partir de uma } \\
\text { aula de ciências que tiveram } \\
\text { sobre o Sistema Solar. Um } \\
\text { dos componentes do grupo } \\
\text { questionou em que a Lua } \\
\text { interfere na Terra. Após } \\
\text { conversas no grupo, ficaram } \\
\text { imaginando o que aconteceria } \\
\text { com a Terra se ela tivesse } \\
\text { duas Luas. }\end{array}$ & $\begin{array}{l}\text { Estudar as influências físicas } \\
\text { da Lua na Terra e projetar o } \\
\text { que aconteceria se fossem } \\
\text { duas Luas. Explorar o } \\
\text { conceito de gravidade. } \\
\text { A partir de conhecimentos } \\
\text { iniciais sobre a Lua, os } \\
\text { estudantes exploraram } \\
\text { também comparações Terra- } \\
\text { Lua em relação a velocidades } \\
\text { e atrações gravitacionais. }\end{array}$ & $\begin{array}{l}\text { Razão e Proporção (a } \\
\text { partir de cálculos relacio- } \\
\text { nados a gravidade); } \\
\text { Conceito de função; } \\
\text { Função quadrática; } \\
\text { Equações do 2 }{ }^{\circ} \text { grau; } \\
\text { fórmula de Bhaskara. }\end{array}$ \\
\hline $\begin{array}{l}\text { Os casos de distúrbios } \\
\text { psicológicos de } \\
\text { antigamente eram } \\
\text { maiores ou menores que } \\
\text { os atuais? Será que há } \\
\text { algo que influencia estes } \\
\text { distúrbios psicológicos } \\
\text { além de traumas e/ou } \\
\text { mágoas? Será que não } \\
\text { tem mais alguma coisa } \\
\text { envolvida? }\end{array}$ & $\begin{array}{l}\text { Os estudantes imaginavam } \\
\text { que a quantidade de } \\
\text { aparelhos eletrônicos e outras } \\
\text { tecnologias dos dias atuais } \\
\text { poderiam afetar de alguma } \\
\text { forma as pessoas e talvez } \\
\text { gerar problemas psicológicos. }\end{array}$ & $\begin{array}{l}\text { Estudar os efeitos invisíveis } \\
\text { dos aparelhos eletrônicos no } \\
\text { nosso meio, tais como as } \\
\text { ondas eletromagnéticas e os } \\
\text { campos eletromagnéticos }\end{array}$ & $\begin{array}{l}\text { Trigonometria: seno, } \\
\text { cosseno e tangente }\end{array}$ \\
\hline
\end{tabular}

Tabela 1: Projetos de Aprendizagem dos estudantes e suas aprendizagens de matemática

Observa-se que Tabela 1 indica em primeiro lugar (colunas 1 e 2) as perguntas inicias dos Projetos de Aprendizagem de cada grupo de estudantes e o contexto dessas curiosidades. Os dados resumidos nessas duas primeiras colunas trazem os subsídios que permitiram aos professores estabelecerem relações com matemática, projetarem possíveis aprendizagens dos estudantes e planejarem suas intervenções. Evidencia-se, desse modo, que o trabalho realizado possibilitou que os estudantes partam do seu real subjetivo (seu contexto de curiosidades e construções prévias) em direção às suas necessidades de compreensão, expressas pelas perguntas iniciais. Destaca-se, ainda, que as perguntas iniciais, bem como as demais curiosidades expressas pelos estudantes, evidenciam ao professor as perturbações e os desequilíbrios cognitivos dos mesmos, os quais, em acordo com a teoria piagetiana, são responsáveis por desencadear os processos de construção do conhecimento pelo sujeito.

$\mathrm{Na}$ terceira coluna, apresentamos sinteticamente as relações planejadas pelos professores e as relações efetivamente estabelecidas pelos estudantes ao longo das investigações e intervenções do tipo 2 realizadas. Nesta perspectiva é interessante 
observar que as trajetórias de aprendizagem dos estudantes podem e devem ser vislumbradas antecipadamente pelo professor, no entanto os caminhos efetivamente percorridos podem ser alterados (como vemos nos Projetos de Aprendizagem relacionados ao congelamento de corpos e a duas Luas). Essa perspectiva é explicada pela possibilidade de escolha e tomada de decisão dos estudantes, que, motivadas pelos seus desequilíbrios cognitivos, levam a ações que se coordenam na busca por uma melhor compreensão que possibilita um novo equilíbrio (equilibração majorante). Nesse sentido, observa-se, ainda, que a possibilidade de alteração de trajetórias é compatível à estruturação de uma escola da Cultura Digital, tendo em vista que nela o estudante deve ter um papel ativo, próprio de um sujeito que administra suas aprendizagens ao enfrentar situações complexas, desafiadoras e subjetivamente inéditas.

Por fim, a quarta coluna indica as aprendizagens de matemática construídas pelos estudantes e que, a partir da intervenção inicialmente comentada sobre o compartilhamento de aprendizagens entre os grupos, constitui a organização curricular de matemática desenvolvida pela turma no período da experiência. Dessa forma, na quarta coluna apresenta-se um conjunto de conceitos de matemática que foram construídos a partir da investigação dos estudantes e efetivou-se como um trabalho que formou uma rede.

\section{CONSIDERAÇÕES FINAIS E PERSPECTIVAS DE INVESTIGAÇÃO}

As discussões teórica e prática pertinentes à investigação da intervenção do professor permitiram indicar a viabilidade do desenvolvimento de Projetos de Aprendizagem como uma estratégia pedagógica promotora de aprendizagens de matemática por estudantes do $9^{\circ}$ ano do Ensino Fundamental. Sustentados, então, pela Epistemologia Genética, os Projetos de Aprendizagem podem ser caracterizados como uma intervenção do tipo 1.

Complementares a este estudo, levantamos duas perspectivas de investigação que se apresentam como necessárias: (i) a discussão sobre os caminhos que levaram os estudantes à construção dos conceitos de matemática e o papel do professor nesse processo e (ii) a extensão da experiência para os quatro anos finais do Ensino Fundamental, de modo a vislumbrar possíveis trajetórias curriculares de matemática desenvolvidas a partir de investigações em matemática advindas de Projetos de Aprendizagens.

Observamos, por fim, a relevância do trabalho para os estudantes, na perspectiva de estabelecerem novas relações com a disciplina de matemática, a qual pôde ser estudada do ponto de vista do jovem, em um efetivo diálogo com a sua realidade cognitiva e exploração da autoria e criatividade no uso das tecnologias digitais e na construção de suas trajetórias de aprendizagens.

\section{REFERENCIAS}

ARRUDA, E. P. Ciberprofessor: Novas Tecnologias, Ensino e Trabalho Docente. Belo Horizonte: Autentica, 2004. 
BASSO, M. V. A. Espaços de Aprendizagem em Rede: novas orientações na formação de Professores de Matemática. Porto Alegre: UFRGS, 2003. 412 f. Tese (Doutorado em Informática na Educação) - PPGIE, UFRGS, Porto Alegre, 2003.

BONA, A. S. D. Espaço de Aprendizagem Digital da Matemática: o aprender a aprender por cooperação. Porto Alegre: UFRGS, 2012, 248 f. Tese (Doutorado em Informática na Educação) - PPGIE, UFRGS, Porto Alegre, 2012.

BONA, A. S. D. Portfólio de Matemática: um instrumento de análise do processo de aprendizagem. Porto Alegre: UFRGS, 2010, 404 f. Dissertação (Mestrado em Ensino de Matemática) - PPGEMat, Instituto de Matemática, UFRGS, Porto Alegre, 2010.

BONA, A. S. D.; MORAIS, A. D.; BASSO, M. V. A.; FAGUNDES, L. C. Cultura Digital e Aprendizagem Cooperativa. In: RENOTE - Revista Novas Tecnologias na Educação, v.10, n.1, 2012.

D'AMBROSIO, U. Educação Matemática: da teoria a práxis. Coleção Perspectivas em Educação Matemática. Campinas, São Paulo: Papirus, 1996.

FAGUNDES, L. C.; NEVADO, R. A.; BASSO, M. V. A. Projeto Escola Conectividade e Sociedade da Informação e do Conhecimento. Porto Alegre, 2001. Disponível em:

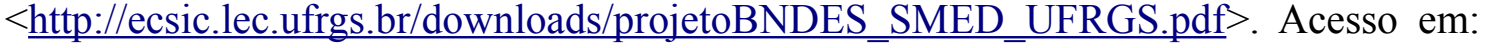
14 set. 2010.

FAGUNDES, L. C.; SATO, L. S.; MAÇADA, D. L. Aprendizes do Futuro: as inovações começaram! MEC, 1999.

FREIRE, P. Pedagogia da Autonomia: saberes necessários à prática educativa. 22 ed. São Paulo: Paz e Terra, 1996.

HOFFMANN, D. S.; FAGUNDES, L. C. Cultura Digital na Escola ou Escola na Cultura Digital? RENOTE - Revista Novas Tecnologias na Educação, Porto Alegre, v. 6, n. 2, 2008.

LEMOS, A. Cibercultura: tecnologia e vida social na cultura contemporânea. Tradução de Carlos Irineu da Costa. Porto Alegre: Sulina, 2002.

LINS, R. C. Matemática, monstros, significados e Educação Matemática. In: BICUDO, M. A. V. e BORBA, M. C. (orgs.). Educação Matemática: Pesquisa em Movimento. São Paulo: Cortez, 2004. P. 92-120.

MATTOS, E. B. V. Construção de conceitos de matemática via Projetos de Aprendizagem. Porto Alegre: UFRGS, 2010, 245 f. Dissertação (Mestrado em Ensino de Matemática) - PPGEMat, Instituto de Matemática, UFRGS, Porto Alegre, 2010.

MATTOS, E. B. V.; BASSO, M. V. A. Projetos de Aprendizagem: uma alternativa aos desafios educacionais do século XXI. In: II Congresso Nacional de Educação Matemática e IX Encontro Regional de Educação Matemática, 2011, Ijuí. Anais. Ijuí: Ed. Unijuí, 2011.

MATTOS, E. B. V.; BONA, A. S. D.; BASSO, M. V. A.; FAGUNDES, L. C. Iniciação Científica e a Aprendizagem de Matemática na Educação Básica. Revista Iberoamericana de Tecnología en Educación y Educación en Tecnología. No prelo. MORAIS, A. D.; FAGUNDES, L. C. A inclusão digital da Escola ou a inclusão da Escola na cultura digital? Diálogo - Ética e Educação, Canoas, n. 19, p. 97-113, jul./dez. 2011.

PIAGET, J. A Equilibração das Estruturas Cognitivas: problema central do desenvolvimento. Tradução de Marion Merlone dos Santos Penna. Rio de Janeiro: Zahar, 1976.

PIAGET, J. Fazer e Compreender. Tradução de Christina Larroudé de Paula Leite. São Paulo: Melhoramentos, 1978. 
PIAGET, J. A Tomada de Consciência. Tradução de Edson Braga de Souza. São Paulo: Melhoramentos, 1977.

RAMOS, E. M. F., et al. Curso de especialização em educação na cultura digital: documento base. Brasília: Ministério da Educação, 2013.

ROSA, M. B. A inclusão da instituição escola na cultura digital e a construção de novos paradigmas a partir da iniciação científica na educação básica. Porto Alegre: UFRGS, 2013, 252 f. Tese (Doutorado em Informática na Educação) - PPGIE, UFRGS, Porto Alegre, 2013.

VEEN, W.; VRAKKING, B. Homo Zappiens: educado na era digital. Tradução de Vinícius Figueira. Porto Alegre: Artmed, 2009. 